\title{
Industry-academia collaborations in software engineering
}

\author{
An empirical analysis of challenges, patterns and anti-patterns in research projects
}

\author{
Vahid Garousi* \\ SnT center, University of \\ Luxembourg, Luxembourg \\ Dept. of Computer Engineering, \\ Hacettepe University, Ankara, Turkey \\ garousi@svv.lu
}

\author{
Michael Felderer \\ Department of Computer Science \\ University of Innsbruck \\ Innsbruck, Austria \\ michael.felderer@uibk.ac.at
}

\author{
João M. Fernandes ${ }^{\dagger}$ \\ Dept. Informática / ALGORITMI \\ Universidade do Minho \\ Braga, Portugal \\ jmf@di.uminho.pt
}

\author{
Dietmar Pfahl $\ddagger$ \\ Institute of Computer Science \\ University of Tartu \\ Tartu, Estonia \\ dietmar.pfahl@ut.ee
}

\author{
Mika V. Mäntylä \\ Faculty of Information Technology \\ and Electrical Engineering \\ University of Oulu \\ Oulu, Finland \\ mika.mantyla@oulu.fi
}

\begin{abstract}
Research collaboration between industry and academia supports improvement and innovation in industry and helps to ensure industrial relevance in academic research. However, many researchers and practitioners believe that the level of joint industry-academia collaboration (IAC) in software engineering (SE) research is still relatively low, compared to the amount of activity in each of the two communities. The goal of the empirical study reported in this paper is to exploratory characterize the state of IAC with respect to a set of challenges, patterns and anti-patterns identified by a recent Systematic Literature Review study. To address the above goal, we gathered the opinions of researchers and practitioners w.r.t. their experiences in IAC projects. Our dataset includes 47 opinion data points related to a large set of projects conducted in 10 different countries. We aim to contribute to the body of evidence in the area of IAC, for the benefit of researchers and practitioners in conducting future successful IAC projects in SE. As an output, the study presents a set of empirical findings and evidence-based recommendations to increase the success of IAC projects.
\end{abstract}

\section{CCS CONCEPTS}

-Software and its engineering $\rightarrow$ Software creation and management; $\bullet$ Social and professional topics $\rightarrow$ Professional topics;

\footnotetext{
*Supported by the National Research Fund, Luxembourg FNR/P10/03.

${ }^{\dagger}$ Supported by FCT (Fundação para a Ciência e Tecnologia) within the Project Scope UID/CEC/00319/2013.

$\ddagger$ Also affiliated with Dept. Electrical and Computer Engineering, University of Calgary, Calgary, Canada. Supported by the institutional research grant IUT20-55 of the Estonian Research Council.
}

Permission to make digital or hard copies of all or part of this work for personal or classroom use is granted without fee provided that copies are not made or distributed for profit or commercial advantage and that copies bear this notice and the full citation on the first page. Copyrights for components of this work owned by others than ACM must be honored. Abstracting with credit is permitted. To copy otherwise, or republish, to post on servers or to redistribute to lists, requires prior specific permission and/or a fee. Request permissions from permissions@acm.org.

EASE'17, Karlskrona, Sweden

(C) 2017 ACM. 978-1-4503-4804-1/17/06 ..\$15.00

DOI: http://dx.doi.org/10.1145/3084226.3084279

\section{KEYWORDS}

software engineering; industry-academia collaborations; research; empirical study; success factors; challenges; patterns; anti-patterns

\section{ACM Reference format:}

Vahid Garousi, Michael Felderer, João M. Fernandes, Dietmar Pfahl, and Mika V. Mäntylä. 2017. Industry-academia collaborations in software engineering. In Proceedings of EASE'17, Karlskrona, Sweden, June 15-16, 2017, 6 pages. DOI: http://dx.doi.org/10.1145/3084226.3084279

\section{INTRODUCTION}

In the software engineering (SE) domain, academia and industry are large communities. Unfortunately, the level of joint industryacademia collaboration (IAC) initiatives in SE is still low. It seems that a SE researcher or a practitioner prefers to interact with fellows from his/her group only [5]. There are many events organized by each of the two communities, but we usually see only handful numbers of participants from the other community in such events. There has been relatively little effort by the two sides to collaborate on joint research activities. Various reasons have been discussed by researchers and practitioners for such a lack of motivation for IACs, such as different objectives for both sides, industrial problems lacking scientific novelty or challenges, and the low applicability and the scalability problems of the solutions developed in the academia $[3,5]$. For the SE research community to have a meaningful future, there is a critical need to better intertwine industry and academia.

The issue of IACs has been an important topic in SE, where industrial relevance and impact of research activities are of utmost importance. For example, there are projects, such as the ACM SIGSOFT Impact project (www.sigsoft.org/impact.html), which have measured and analyzed the impact of SE research on practice. To stress the importance of IAC, to discuss success stories and how to "bridge the gap", various workshops and panels are regularly organized within international research conferences. An example is the panel called "What industry wants from research" at ICSE 2011, in which interesting ideas from companies were presented. Another 
international workshop on the topic of long-term industrial collaborations on software engineering (called WISE) was organized in 2014, which hosted several interesting talks.

A recent Systematic Literature Review (SLR) [5] synthesized the challenges, patterns (best practices, i.e., what to do to ensure success), and anti-patterns (what not to do) in IAC projects. Based on the results of the SLR study, the goal of the study reported in this paper is to characterize IAC projects in SE, w.r.t. the challenges, patterns, and anti-patterns identified by the SLR. To address the above goal, we conducted an opinion survey to gather the data from researchers and practitioners. In summary, this paper contributes with (1) a ranking of the challenges, patterns, and anti-patterns in a large set of international IAC projects (across 10 countries), and (2) evidence-based recommendations to ensure success and to prevent problems in IAC projects.

The remainder of this paper is structured as follows. A review of the related work is presented in Section 2. We describe the study goal, research questions and research methodology in Section 3. Section 4 presents the results. Finally, in Section 5, we draw conclusions, and suggest areas for further research.

\section{RELATED WORK}

As stated above, a recent SLR [5] systematically synthesized the body of literature on the subject of IAC projects in SE (33 papers in this area), which the reader can refer to for details. Through a systematic qualitative coding process based on grounded theory, the SLR derived a list of 64 challenges, 128 patterns and 36 antipatterns for IAC projects. The SLR categorized those challenges, patterns and anti-patterns into 11, 16 and 7 categories, respectively.

Out of the 33 primary studies reviewed in the SLR, while 17 experience reports shared insightful experience and evidence on the topic, we observe that the area still lacks the following types of empirical evidence: (1) most of the experience are reported by focused (single) teams of researchers and practitioners and there is a need for evidence based on a larger, more distributed set of IAC projects to reduce the sampling bias; (2) challenges, success patterns and anti-patterns in IAC projects have been reported rather sparsely and sporadically, and there is a need for more holistic and systematic synthesis of those issues.

The current work is based on a first study [4], which analyzed (both quantitatively and qualitatively) a set of 10 IAC software testing projects conducted in Canada and Turkey w.r.t. challenges, patterns and anti-patterns. As outputs, that study presented a set of empirical findings and evidence-based recommendations. For example, it reported that even if an IAC project may seem to possess all the major conditions to be successful, one single challenge (e.g., disagreement in confidentiality agreements) can lead to its failure. Thus, the study recommended that both academics and practitioners should consider all the challenges early on and pro-actively work together to eliminate the risk of challenges in IAC projects.

Another survey [9] also investigated the success factors for IAC in SE. Overall, 48 researchers and 41 practitioners from Sweden and Australia participated in the survey. The most important lessons from the study are that (1) buy-in and support from company management is crucial, (2) there must be a champion in the company, and not only a person assigned the responsibility, (3) there are different understandings between different categories of people, and (4) social skills are particularly important in a long-term collaboration. Differing from that survey, our data points were not the responding persons, but research projects. Furthermore, our study as presented in this paper is not limited to success factors, but investigates challenges, success patterns and anti-patterns.

Other empirical studies on IAC $[1,2]$ have also been reported in different fields, such as management. For example, the study presented in [1] assesses the most influential factors for success or failure in research projects between university and industry. The study analyzes the factors leading to success or failure, and was based on interviews with 30 university researchers. The study concluded that the company's real interest and involvement during the project, its capacity to assimilate new knowledge, and a confident attitude towards the university researchers were the crucial factors for assuring a successful collaboration.

\section{RESEARCH GOAL AND METHOD}

\subsection{Goal and research questions}

Formulated using the Goal, Question, Metric (GQM) approach [8], the goal of this study is to characterize a set of IAC projects in SE, with respect to the challenges, patterns, and anti-patterns identified by the SLR study [5]. Our study contributes to the body of evidence in the area of IAC, for the benefit of SE researchers and practitioners in conducting successful projects in the future. Based on the above goal, we raised the following two research questions (RQs):

RQ1 To what extent did each challenge, pattern, and antipattern synthesized in the SLR study [5] play a role in the IAC projects under study?

RQ2 What have been the success levels of the IAC projects and what were the correlations of challenges, patterns, and anti-patterns with success measures in those projects?

We adopted the notion of the success criteria as synthesized in the SLR study [5], which are defined quite differently by researchers and practitioners. The academic success criteria are usually the number of resulting publications and the quality of the trained graduate students. The industrial success criteria are usually impact and success of the solutions developed in the project.

\subsection{Research method}

To address the above goal, we conducted an opinion survey to gather the opinions from researchers and practitioners. In designing the survey, we followed the survey guidelines in SE [6].

Table 2 shows the structure of the questionnaire used to conduct the survey. The questionnaire has 30 questions that were asked w.r.t. one single IAC project in which the respondent had participated.

The two RQs of the study were addressed by the questions in parts 3 and 4 of the tab:questionnaire. The entire list of challenges, patterns and anti-patterns were adopted from the SLR study [5] and included as questions in the questionnaire. We asked participants about how frequently each of the challenges were 'observed' using a 5-point Likert scale (0: not observed, 1: somewhat observed, 2: moderately observed, 3: highly observed, and 4: very highly observed). We were also interested in the 'impact' of each challenge also rated a 5-point Likert scale (0: no impact, 1: minor negative impact, 2: moderate negative impact, 
3: high negative impact, and 4: very high negative impact). We asked similar questions to gather data for the impacts of applying each of the 15 types of patterns and committing 4 types of anti-patterns, as listed in Table 1.

Table 1: Categories of patterns and anti-patterns.

\begin{tabular}{lc}
\hline Patterns & \\
Proper and active knowledge management & PAKM \\
Ensuring engagement and managing commitment & ENMC \\
Considering and understanding industry's needs, and & CUIN \\
$\quad$ giving explicit industry benefits & HMRU \\
Having mutual respect, understanding and appreciation & BA \\
Being Agile & WTI \\
Working in (as) a team and involving the "right" practitioners & CMRL \\
Considering and manage risks and limitations & ROSP \\
Researcher's on-site presence and access & FPRM \\
Following a proper research/data collection method & MFRP \\
Managing funding/recruiting/partnerships & UCCL \\
$\quad$ and contracting privacy & ERPM \\
Understanding the context, constraints and language & CMA \\
Efficient research project management & TPS \\
Conducting measurement/ assessment & PTS \\
Testing pilot solutions before using them in industry & \\
Providing tool support for solutions & FSCA \\
\hline Anti-patterns & UDS \\
Following self-centric approach & PCM \\
Unstructured decision structures & IPOP \\
Poor change management & \\
Ignoring project, organizational, or product characteristics & \\
\hline
\end{tabular}

In our opinion survey, the unit of analysis is a single IAC project. Thus, a participant could provide multiple answers; each one for a single project that $\mathrm{s} /$ he had been involved in. The IAC project could be completed or ongoing. The survey was anonymous but the participants could provide their names and emails if they wanted to be contacted for follow-up interviews.

Invitations were sent to the SE researchers who were active in IAC projects, the authors of the studies reviewed in the SLR [5], and also our industrial contact points. The authors of this study are also active in IACs, so they also provided data points from their projects. At the end, we collected a total of 47 opinion data points, from which 36 data points were from the study authors. It was quite unfortunate to receive a small ratio of data points from outside the authors' team as only about $25.5 \%$ ( 11 out of 43 ) of the invited researchers and practitioners provided data points.

Our sampling method was 'convenience sampling'. As reported in a highly-cited survey on controlled experiments in SE [7], convenience sampling is the dominant survey and experimental approach in SE. Albeit its drawbacks and bias in the data, this does not mean that convenience sampling is generally inappropriate. Convenience sampling is also common in other disciplines such as clinical medicine and social sciences.

\section{RESULTS AND ANALYSIS}

We first present a brief overview of demographics of the dataset. We then analyze the data and answer each of the RQs.

\subsection{Demographics}

One of the questions asked about the respondent's affiliation and position: of 47 data points, 42 were provided by university academics (researches), 4 by practitioners (from industry), and 1 from a fellow in a large research center. Another question asked about the country (or countries) in which the IAC project was conducted. Figure 1 shows that 10 countries are represented in the dataset.

Table 2: Structure of the questionnaire used for the survey.

\begin{tabular}{lc}
\hline part & $\begin{array}{c}\text { number of } \\
\text { questions }\end{array}$ \\
\hline 1: Profile of the researcher/project & 12 \\
2: Need, offered solutions, and impact & 3 \\
3: Observed challenges, and applied & 8 \\
$\quad$ patterns and anti-patterns & 5 \\
4: Outcome and success criteria & 2 \\
5: Any other related feedback & 30 \\
Total & \\
\hline
\end{tabular}

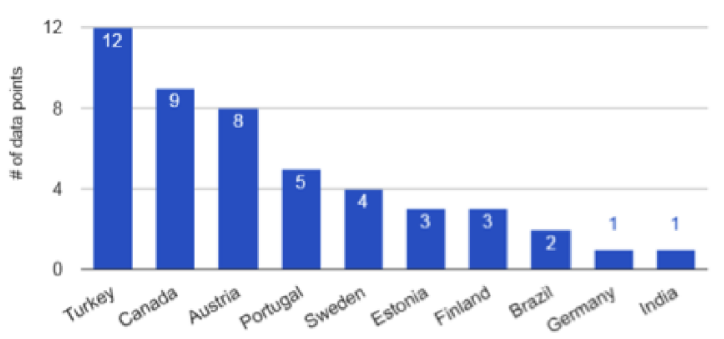

Figure 1: Breakdown of the dataset by countries.

As shown in Table 2, part 2 of the survey queried about needs, solutions offered, and industrial impacts of the IAC project. Table 3 shows the considered categories of challenges and their abbreviations. We were also interested in the SE topics of the projects in the dataset. To classify the SE topics, we used version 3.0 of

Table 3: Categories of challenges (adopted from the SLR [5]).

\begin{tabular}{lc}
\hline Lack of research relevance & LRR \\
Problems associated with the research method & RM \\
Lack of training, experience, and skills & LTES \\
Lack or drop of interest / commitment & LDRC \\
Mismatch between industry and academia & MIA \\
Communication-related challenges & CRC \\
Human and organizational challenges & HOC \\
Management-related challenges & MRC \\
Resource-related challenges & RRC \\
Contractual and privacy concerns & CPC \\
\hline
\end{tabular}


the Software Engineering Body of Knowledge (SWEBOK), which includes 12 Knowledge Areas (KAs). We did not have a specific question about this, but we easily derived the SE topics of an IAC project by looking at the need/problem tackled. Figure 3 shows the breakdown of the data points by the SE topics. Testing is the most frequent topic in the dataset. There was no opinion for any project in the 'professional practice' knowledge areas of the SWEBOK.

\subsection{RQ1: Frequency of challenges, patterns and anti-patterns in the IAC projects}

Figure 2 shows in a scatter plot the average observation frequencies of the challenges and the level of their negative impact on IAC projects. As one would expect, the types and observation frequencies of challenges and their impact vary from project to project.

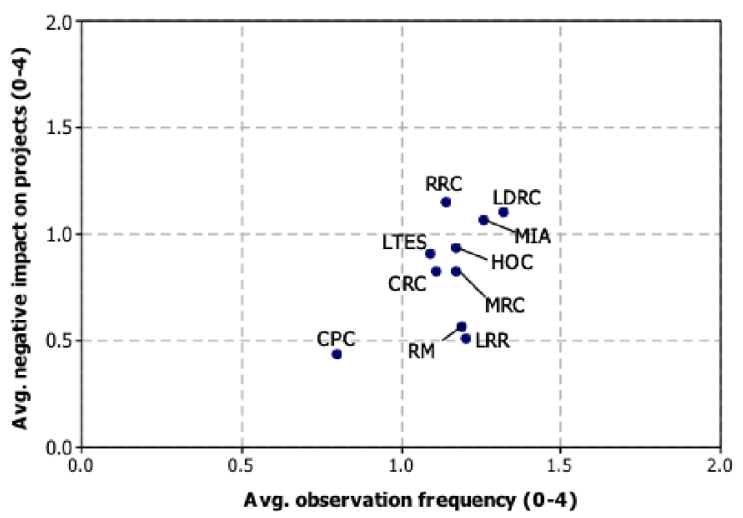

Figure 2: Observed frequencies of the challenges and extents of their negative impacts on projects.

In general, over the sample of projects, the challenges were typically only somewhat or moderately observed. This relatively-low level of challenges could denote the high relative maturity/expertise of most of the respondents in the context of the reported IAC projects. But still, some respondents reported that they highly or very highly observed some of the challenges, as shown in Fig. 2. On average, the three most frequent challenges are lack or drop of interest / commitment (LDRC, average $=1.32$ ), mismatch between industry and academia (MIA, average $=1.26$ ), and lack of research relevance $(\mathrm{LRR}$, average $=1.20$ ). The challenge with the lowest average frequency is contractual and privacy concerns (CPC, 0.8). The three challenges with an average impact above 1.0, i.e., minor negative impact, are resource-related challenges ( $R R C$, average $=1.2$ ), lack or drop of interest / commitment (LDRC, 1.11), as well as mismatch between industry and academia (MIA, 1.09). The challenge with the lowest impact is again contractual and privacy concerns (CPC).

The Pearson correlation coefficient between the observed average frequencies and the average negative impacts of the challenges is 0.56 , with the p-value of 0.09 . That means that a slightly positive slightly-significant correlation exists, i.e., the more a given challenge is observed, the higher negative impact it has on a project.

To assess the data in more detail, Fig. 4 shows the histogram of all the frequencies (and average values) of the challenges. The median values of all the distributions are equal to 1 . All the histograms are skewed towards the left, which means that the observation frequencies of the challenges were reported to be low in general. This has been perhaps due to experience and expertise of the participants in carrying out IAC projects.

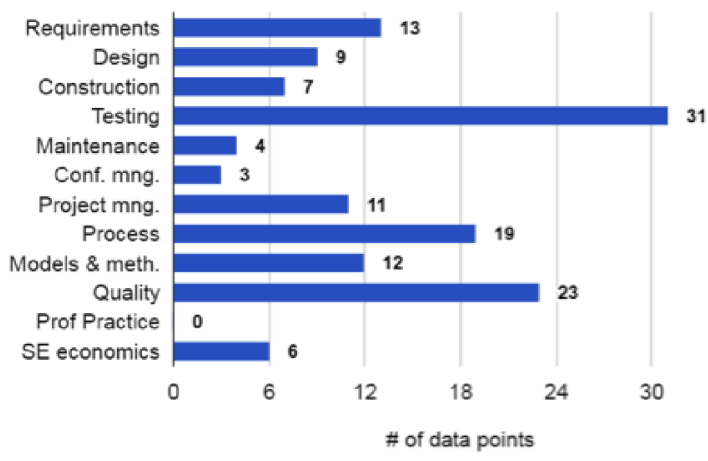

Figure 3: Breakdown of data points by the SE topics.

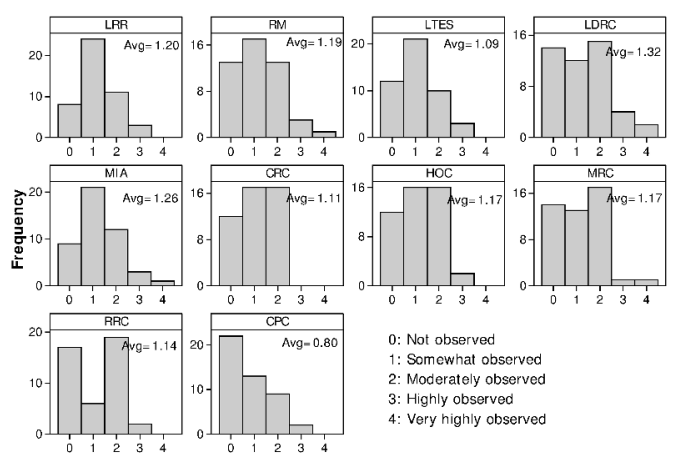

Figure 4: Histogram of all the reported frequencies of the challenges.

We also measured the impacts of applying "patterns" (what to do to ensure success) [5] and committing anti-patterns (what not to do). Figure 5 shows the average values of the reported data. The interpretation of each scale point $[-2,2]$ is also shown in Fig. 5. The three practices with the highest positive impact values are: (1) Having mutual respect, understanding and appreciation (HMRU, average $=1.33$ ), (2) Working in (as) a team and involving the "right" practitioners (WTI, average $=1.31$ ), and (3) Testing pilot solutions before using them in industry (TPS, average $=1.26$ ). In terms of anti-patterns (the last four practices in Figure 11), the participants reported negative impacts for them, as expected. The anti-pattern with the highest negative impact is "Following self-centric approach" (FSCA) in conducting IAC projects, i.e., each side (industry and academia) focuses only on its needs and style in the project.

\subsection{RQ2: Success levels and correlations with challenges, patterns and anti-patterns}

Two questions of the survey were related to the success levels of each IAC project. As discussed in Section 3.1, We adopted the notion of the success criteria as synthesized in the SLR study [5]. 
Figure 6 shows the results and also the average and the median values. The average values of the two datasets are quite similar, i.e., 2.81 and 2.70 (out of 4), denoting that the IAC projects in the dataset had higher-than-average success levels. Four and six projects were reported to have poor success levels equal to 0 and 1 , respectively, from academic and industrial perspectives.

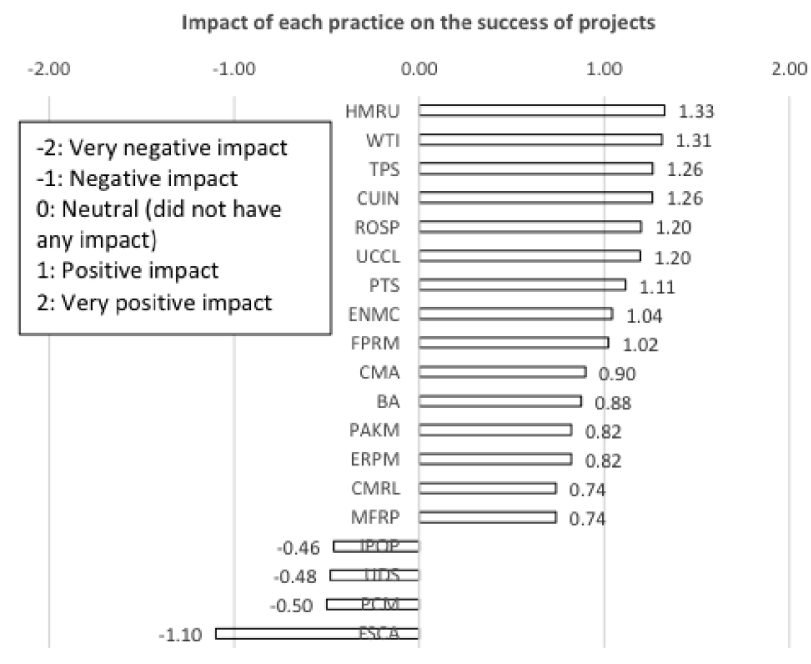

Figure 5: Reported impacts of patterns and anti-patterns on projects (average values).

The Pearson correlation coefficient between the success levels from academic and industrial perspectives is 0.49 with $\mathrm{p}$-value $=$ 0.001 , denoting that there is a positive correlation of high significance, i.e., the higher the academic success of a given project, the higher its expected industrial success would be. Figure 7 shows, as a bubble plot, the pair-wise numbers of success levels. Most of the data points are in the top-right corner of this bubble plot. There are also some data points in the "extreme", for example, a project had a success level of 3 from the academic perspective, but was only at a success level of 1 from the industrial perspective. In general, it seems also that the IAC projects are slightly more successful from the academic side than from the industrial side, as there are bigger bubbles below the diagonal that above it.

Pearson correlation coefficients and $\mathrm{p}$-values are shown in Table 4 . The Pearson correlation coefficients for the datasets in these two datasets are quite low ( -0.09 and -0.12$)$. We were expecting higher negative correlations, i.e., the higher the level of challenges in a given project, the lower its success is expected to be.

Table 4 lists the various correlation analyses. The last column shows the correlations between sum of patterns with the total challenges impact as well as sum of anti-patterns with the total challenges impact. All correlations, but the one between academic and industrial success measures, are low and not significant.

\subsection{Lessons learned and recommendations}

Based on the results of the empirical study, this paper offers three experience-based messages (best practices), that when applied, should ensure success in IAC projects. They are titled as: "common goal", "understanding and team work", and "managerial topics".

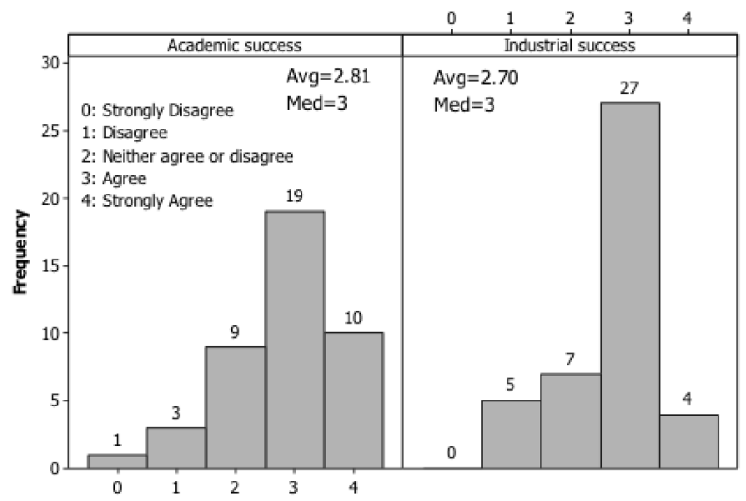

Figure 6: Success levels of projects, from academic and industrial perspectives.

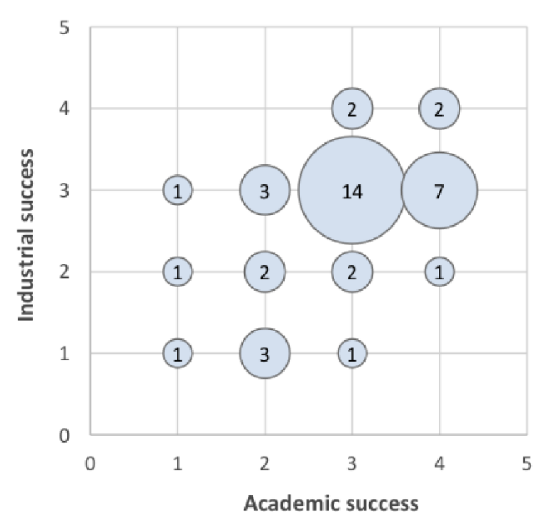

Figure 7: Pair-wise numbers of project success levels.

Table 4: Pearson correlation coefficients and p-values.

\begin{tabular}{lcccccc}
\hline & \multicolumn{2}{c}{$\begin{array}{c}\text { Academic } \\
\text { success }\end{array}$} & \multicolumn{2}{c}{$\begin{array}{c}\text { Industrial } \\
\text { success }\end{array}$} & \multicolumn{2}{c}{$\begin{array}{c}\text { Total challenges } \\
\text { impact }\end{array}$} \\
& Corr. & p-value & Corr. & p-value & Corr. & p-value \\
\hline $\begin{array}{l}\text { Industrial } \\
\text { success }\end{array}$ & +0.42 & 0.00 & & & & \\
\hline $\begin{array}{l}\text { Total challenges } \\
\text { impact }\end{array}$ & -0.09 & 0.55 & -0.12 & 0.42 & & \\
\hline $\begin{array}{l}\text { Sum of } \\
\text { patterns }\end{array}$ & +0.11 & 0.45 & +0.16 & 0.29 & -0.35 & 0.01 \\
\hline $\begin{array}{l}\text { Sum of } \\
\text { anti-patterns }\end{array}$ & -0.03 & 0.84 & -0.09 & 0.55 & -0.14 & 0.34 \\
\hline
\end{tabular}

A "common goal" is a cornerstone of any IAC project. Figure 4 shows how the lack of interests or drop of interest is the challenge with the highest perceived negative impact. Mismatch between industry and academia is the challenge with the second highest adverse impact. Both of these challenges reflect that a common goal for the research project is missing. Without a common goal, a IAC project would often have a little chance of success. 
"Understanding and team work" is related to the above pattern ("common goal"), as it ensures that the IAC project progresses smoothly and the common project goal is not lost throughout the project. Usually, practitioners and researchers have different cultures, backgrounds, and objectives. Thus, it is not a surprise that all of the top-3 ranked success criteria deal with the topic of gaining understanding and forming a team: first "Having mutual respect, understanding and appreciation", second "Considering and understanding industry needs", and third "Working as a cohesive team".

Even when a common goal exists and a team with a mutual understanding has been formed, an IAC project can fail due to management-related issues. For instance, contractual and privacy concerns need to be considered. Getting and keeping higher levels of management commitment is important, as otherwise top-level mangers can abruptly shut-down IAC projects if they think the company employees are wasting their time without a clear benefit to the company. Internal company policies need to be understood; for example, some units or sites of a large company may not have permission to be involved in research activities. Some of these managerial topics could be impossible to bypass.

\subsection{Limitations and threats to validity}

In this section we discuss threats to internal, construct, conclusion, and external validity as well as measures to mitigate them.

Internal validity: It is a property of scientific studies which reflects the extent to which a causal conclusion based on a study and the extracted data is warranted. A potential problem with internal validity (cause-effect) is that the problems observed are purely based on the opinions of the participants (observers). They believed they have observed effects that caused problems (or success), but it may not be the case in reality. We did what is typical: we counted the votes for each question and then made statistical inferences. The results based on such voting data reflects the opinions of the participants who participated in the study. It is also common for people to deflect their answers when they feel being evaluated and based on what they think is the intended result of a study. To mitigate this, we informed participants prior to the survey that that participants will remain anonymous.

Construct validity: Construct validity is about how well the measured variables represent the constructs (concepts) under investigation. In a questionnaire-based study like ours, typically several items (questions) form one construct. We showed the mapping of the questionnaire questions to the constructs under investigation in Table 2 and discussed the rationale behind them in Section 3.2.

Conclusion validity: Conclusion validity of a study deals with whether correct conclusions are reached through rigorous and repeatable treatment. We analyzed, qualitatively, challenges and success criteria of IAC projects. For each RQ, we attempted to reduce the bias by seeking support from a 5-point Likert scale data, gathered in the survey, and statistical results. All the conclusions that we present in this paper are strictly traceable to data.

External validity: External validity is concerned with the extent to which the results of this study can be generalized. A threat to external validity in this study lies could be the selection bias (i.e., randomness of the projects participating in our survey) and the moderate number of 47 projects included in the analysis. 36 of the data points ( $76 \%$ of the data) were from the study authors. While we had aimed at gathering a wider and more general survey dataset, the nature of dataset turned out to lead the paper to be more like an experience report of the authors than a general survey. However, as discussed in the data collection phase, we included data points from various types of IAC projects, various domains, and from various countries. Thus, although the dataset is not very large, we think that it is quite diverse in nature and meaningful conclusions can be drawn. Also, due to low participation of practitioners (only 4 data points), the views are basically those of academics. Last but not least, since we used the convenience sampling, there could be a problem with generalization to the target population (IACs in SE and their stakeholders).

\section{CONCLUSIONS AND FUTURE WORK}

This paper reported a survey on IACs in software engineering. Our results are based on 47 different projects from 10 different countries, and covering 11 out of 12 knowledge areas of the SWEBOK. The results show that lack or drop of interest / commitment (LDRC) is the most highly observed challenge in the projects. The challenge with the highest perceived negative impact is resource-related challenges (RRC) followed closely by LDRC. Unexpectedly, our statistical analysis shows that perceived challenges are not correlated with project success or failure. In order to ensure success in IAC projects, we suggest focusing on three issues: (1) finding and maintaining a common goal; (2) focusing on mutual understanding (between academia and industry) and teamwork; and (3) making sure that managerial topics do not prevent project success. As future work, we plan to replicate this study with more data points from a larger set of projects. We would like to use the findings from this study w.r.t. challenges, patterns and anti-patterns in our next IAC projects. We also plan to conduct more in-depth analysis of the dataset using the "case survey" research method.

\section{REFERENCES}

[1] Ana M. Bernardos Barbolla and José R. Casar Corredera. 2009. Critical factors for success in university-industry research projects. Technology Analysis \& Strategic Management 21, 5 (2009), 599-616.

[2] Tina Barnes, Ian Pashby, and Anne Gibbons. 2002. Effective university-industry interaction: A multi-case evaluation of collaborative R\&D projects. European Management fournal 20, 3 (2002), 272-285.

[3] Lionel C. Briand. 2012. Embracing the engineering side of software engineering. IEEE Software 29, 4 (2012), 96.

[4] Vahid Garousi, Matt M. Eskandar, and Kadir Herkiloğlu. 2016. Industry-academia collaborations in software testing: Experience and success stories from Canada and Turkey. Software Quality fournal (2016), 1-53.

[5] Vahid Garousi, Kai Petersen, and Baris Ozkan. 2016. Challenges and best practices in industry-academia collaborations in software engineering. Information and Software Technology 79 (2016), 106-127.

[6] Jefferson Seide Molléri, Kai Petersen, and Emilia Mendes. 2016. Survey guidelines in software engineering: An annotated review. In 10th ACM/IEEE International Symposium on Empirical Software Engineering and Measurement (ESEM 2016). Article 58

[7] Dag I. K. Sjoberg, Jo E. Hannay, Ove Hansen, Vigdis By Kampenes, Amela Karahasanovic, Nils-Kristian Liborg, and Anette C. Rekdal. 2005. A survey of controlled experiments in software engineering. IEEE Transactions on Software Engineering 31, 9 (2005), 733-753.

[8] Rini van Solingen and Egon Berghout. 1999. The Goal/Question/Metric method: A practical guide for quality improvement of software development. McGraw-Hill.

[9] Claes Wohlin, Aybüke Aurum, Lefteris Angelis, Laura Phillips, Yvonne Dittrich, Tony Gorschek, Håkan Grahn, Kennet Henningsson, Simon Kågström, Graham Low, Per Rovegard, Piotr Tomaszewski, Christine Van Toorn, and Jeff Winter. 2012. The success factors powering industry-academia collaboration. IEEE Software 29, 2 (2012), 67-73. 